NBER TECHNICAL WORKING PAPER SERIES

SPECIFICATION TESTING IN PANEL DATA WITH INSTRUMENTAL VARIABLES

Gilbert E. Metcalf

Technical Working Paper No. 123

\author{
NATIONAL BUREAU OF ECONOMIC RESEARCH \\ 1050 Massachusets Avenue \\ Cambridge, MA 02138 \\ June 1992
}

My thanks to Whitney Newey, Gregory Chow and the referees for helpful advice. This paper is part of NBER's research program in Public Economics. Any opinions expressed are those of the author and not those of the National Bureau of Economic Research. 
NBER Technical Working Paper \#123

June 1992

\section{SPECIFICATION TESTING IN PANEL DATA WITH INSTRUMENTAL VARLABLES}

\section{ABSTRACT}

This paper shows a convenient way to test whether instrumental variables are correlated with individual effects in a panel data set. It shows that the correlated fixed effects specification tests developed by Hausman and Taylor (1981) extend in an analogous way to panel data sets with endogenous right hand side variables. In the panel data context, different sets of instrumental variables can be used to construct the test. Asymptotically, I show that the test in many cases is more efficient if an incomplete set of instruments is used. However, in small samples one is likely to do better using the complete set of instruments. Monte Carlo results demonstrate the likely gains for different assumptions about the degree of variance in the data across observations relative to variation across time.

Gilbert E. Metcalf

Department of Economics Princeton University

Princeton, NJ 08544-1021 and NBER 


\section{Introduction}

The use of panel data sets has increased dramatically since the pioneering research of Mundlak (1961), Nerlove (1971) and Maddala (1971), among others. An important benefit of pooled cross section and time series data is the possibility of controlling for unobservable individual specific effects. If these unobserved varlables are correlated with right hand side variables in the regression, ordinary least squares (OLS) estimates of the coefficients will be biased and inconsistent. In the presence of correlated individual effects, first difference or fixed effects (within) estimators yield consistent estimates of the regression parameters. However consistency comes at a cost: ignoring the between groups information may substantially reduce the efficiency of the estimates. As a result, a good deal of research has been undertaken to derive tests to detect this possible correlation (e.g. Hausman (1978), Hausman and Taylor (1981), Chamberlain (1983), and Holtz-Eakin $(1988))$

However, none of the tests allow for the possibility that some of the right hand side variables are correlated with the random error (aside from the individual effect). This is perhaps not surprising. Little work has been done on estimation of panel data models in a simultaneous system. ${ }^{1}$ Below, I extend the results of Hausman (1978) and Hausman and Taylor (1981) to the case where right hand side variables are assumed to be endogenous (specifically, correlated with the time varying component of the error structure). It turns out that the IV analogous specification tests for correlated fixed effects given in Hausman and Taylor (1981) are applicable in this context. However,

1 Cornwell, Schmidt and Wyhowski (1991) review the limited literature and provide results which extend the results from the single equation literature in a limited information context (2SLS) to a full information context ( $3 S L S$ ). They do not discuss the issue of specification testing in the context of instrumental variable estimation. 
It is important to specify the instrument set appropriately for the specification test. I then consider the small sample properties of the test statistic under different assumptions about the quality of the instrument and the degree of correlation between the fixed effects and the instrument. Perhaps surprisingly, the appropriate test statistic in many cases uses an inefficient estimator. Asymptotically, while the variance used to construct the test statistic will be greater than the variance associated with using a more efficient estimator, its asymptotic bias will also be greater as the null hypothesis of no correlation is violated. The increase in bias more than offsets the increase in variance thereby leading to a more powerful test statistic.

The degree to which the test statistic using an inefficient estimator is an improvement over the statistic using the efficient estimator depends on the relative amounts of the variance of the explanatory variables and the Instruments which is due to variation across individuals versus across time (the "between" versus the "within" variation). If the ratio of the variance components is the same for the explanatory variables and the instruments, then the two test statistics are equally powerful. However, in small samples the test statistic using the more efficient estimator often performs better as I show below.

The next section shows that the test statistic as suggested by Hausman and Taylor (1981) carries over to the 2SLS case. I discuss the appropriate construction of the instrument set given various assumptions about the type of correlation between the instruments and the individual effects. The following section presents results from a simple Monte Carlo experiment. Finally there is a brief conclusion. 


\section{The Model and Test}

The model under consideration is

$$
Y-X \beta+\alpha \otimes e_{T}+\epsilon
$$

where $Y$ is an NT $X 1$ vector, $X$ an NT $x k$ matrix, $\alpha$ an $N \times 1$ vector of individual effects $\left(\alpha_{1}\right.$ ild with mean 0 and variance $\left.\sigma_{\alpha}^{2}\right)$ and $\epsilon$ an iid random vector with mean 0 and covariance matrix $\sigma_{\epsilon}^{2} I_{N I}$. The vector $e_{T}$ is a $T \times 1$ vector of ones. The data are stacked by individuals over time. That is, $Y^{\prime}-$ $\left[Y_{1}^{\prime} Y_{2}^{\prime} \ldots Y_{N}^{\prime}\right]$ where $Y_{1}$ is a $T \times 1$ vector of observations on the $i^{\text {th }}$ individual. This equation is part of a simultaneous system and by assumption some columns of $X$ are correlated with $\epsilon$. It is assumed that some (possibly ali) columns of $X$ are also correlated with the individual effects. There is a set of instruments 2 , a matrix NT $\times L, L \geq k$, valid in the sense that $Z$ is correlated with $X$ but uncorrelated with $\epsilon$. It is assumed that columns of $X$ which are uncorrelated with 6 are contained in $Z$. The present purpose is to test whether $z$ is correlated with the individual effects. Specifically, I consider the hypotheses:

$$
\begin{aligned}
& H_{0}: \operatorname{plim}_{N \rightarrow \infty}^{N}\left(\sum_{1=1}^{N} Z_{1 t}^{\prime} \alpha_{1} / N\right)-0 \\
& H_{A}: \operatorname{plim}_{N \rightarrow \infty}^{N}\left(\sum_{1=1} Z_{1 t}^{\prime} \alpha_{1} / N\right)-0
\end{aligned}
$$

An alternative null hypothesis which appears less restrictive is that $\underset{N \rightarrow \infty}{\lim } \sum_{1=1}^{N} Z_{1}^{\prime} \cdot \alpha_{1} / N$ - Owhere $Z_{1}$ is the average over time of the observations of $Z_{1 t}$. However, Amemiya and McCurdy (1986) note that the two sets of assumptions are equivalent if one also assumes that the estimator for $\beta$ continues to be consistent when estimated using any $T-1$ of the $T$ time periods. While there may be circumstances in which this second set of T-l assumptions fails to hold while the assumption that plim $\sum_{1=1}^{N} z_{1}^{\prime} \cdot \alpha_{i} / N=0$ holds, it seems reasonable to believe this is an unusual case. Hence I argue that for our purposes the null hypothesis as constructed above is not overly restrictive. 
Given the loss of information resulting from the use of the within or first difference estimators to eliminate correlated fixed effects, there is a large gain possible if one can assume the null hypothesis. In this case, the GLS-IV estimator will be an improvement. Letting $\mathrm{u}=c \otimes \mathrm{e}_{\mathrm{T}}+\epsilon$, then

$$
E\left(u u^{\prime}\right)=\Omega=T \sigma_{\alpha}^{2} P_{v}+\sigma_{\epsilon}^{2} I_{N I}
$$

or

where $\sigma_{1}^{2}=T \sigma_{\alpha}^{2}+\sigma_{\varepsilon}^{2}, P_{V}=\left(I_{N} \otimes e_{T} e_{T}^{\prime}\right) / T$ and $Q_{V}=I-P_{V}$, For future reference, I use the fact that $\Omega^{-1 / 2}=\sigma_{1}^{-1} P_{v}+\sigma_{\epsilon}^{-1} Q_{v}$ and denote $\Omega^{-1 / 2}$ by $H$. $P_{v} X$ replaces the observations for each column of $x$ by the average of the observations for each individual over time. $Q_{v} X$ replaces the observations by the deviations from the time averages.

First note that the Hausman type specification test comparing the GLS-IV estimator with the fixed effects (within) estimator can be constructed using the within and the between estimators. Define the operator $A^{\pi}$ as the projection operator: $A^{\pi}-A\left(A^{\prime} A\right)^{-1} A^{\prime}$. If $Z$ is a set of variables uncorrelated with $\epsilon$, there are different possible instrument sets that I can use, Following the general approach of Cornwel1, Schmidt and Wyhowski, I consider instrument sets of the form $\bar{Z}=\left[Q_{v} Z, P_{v} B\right]$ where $B$ is defined as a matrix of potential instruments. The GLS-IV estimator is given by

$$
\hat{\beta}_{\mathrm{GLS}}^{\mathrm{IV}}=\left(\mathrm{X}^{\prime} \mathrm{H}^{\prime} \overline{\mathrm{Z}}^{\pi} \mathrm{HX}\right)^{-1} \mathrm{X}^{\prime} \mathrm{H}^{\prime} \overline{\mathrm{Z}}^{\pi} \mathrm{HY} \text {. }
$$

Some simple algebra shows that the GLS-IV estimator is a matrix weighted average of the within IV estimator $\left(\hat{\beta}_{W}^{I V}\right)$ and the between IV estimator $\left(\hat{\beta}_{B}^{I V}\right)$. That is,

$$
\hat{\beta}_{\mathrm{GLS}}^{I V}=\Lambda \hat{\beta}_{W}^{I V}+(I-\Lambda) \hat{\beta}_{\mathrm{B}}^{I V}
$$

where 


$$
\begin{aligned}
& \Lambda=\left[\sigma_{\epsilon}^{-2} X^{\prime}\left(Q_{v} Z\right)^{\pi} X+T \sigma_{1}^{-2} \bar{X}^{\prime} \dot{B}^{\pi} \dot{X}\right]^{-1} \sigma_{\epsilon}^{-2} X^{\prime}\left(Q_{v} Z\right)^{\pi} X \text {, } \\
& \hat{\beta}_{W}^{I V}=\left[X^{\prime}\left(Q_{v} Z\right)^{\pi} X\right]^{-1}\left[X^{\prime}\left(Q_{v} Z\right)^{\pi} Y\right] \text {. } \\
& \hat{\beta}_{\mathrm{B}}^{\mathrm{IV}}-\left[\overline{\mathrm{X}}^{\prime} \overline{\mathrm{B}}^{\pi} \overline{\mathrm{X}}\right]^{-1}\left[\overline{\mathrm{X}} \cdot \overline{\mathrm{B}}^{\pi} \overline{\mathrm{Y}}\right] \text {. }
\end{aligned}
$$

In equations (5) and (7), $\overline{\mathrm{X}}-\left[\overrightarrow{\mathrm{X}}_{1}^{\prime} \dot{\mathrm{X}}_{2}^{\prime} \ldots \overline{\mathrm{X}}_{\mathrm{N}}^{\prime}\right]^{\prime}$ where $\overline{\mathrm{X}}_{1}$ is the mean of the $T$ observations on $X$ for the $i^{\text {th }}$ individual (and similarly for $B, Y$, and 2). That equation (4) holds should not be surprising as it is simply the IV analog to the result for OLS estimators derived in Maddala (1971).

Under the null hypothesis, $\hat{\beta}_{\mathrm{GLS}}^{\text {IV }}$ and $\hat{\beta}_{\mathrm{W}}^{\text {IV }}$ are consistent estimators of $\beta$ with $\hat{\beta}_{\text {GLS }}^{\text {IV }}$ the more efficient estimator while under the alternative, $\hat{\beta}_{W}^{\text {IV }}$ is consistent and $\hat{\beta}_{\mathrm{GLS}}^{\mathrm{IV}}$ is inconsistent. A Hausman test statistic of the form

$$
c=\left(\hat{\beta}_{\mathrm{GLS}}^{\mathrm{IV}}-\hat{\beta}_{\mathrm{W}}^{\mathrm{IV}}\right) \cdot \mathrm{V}\left(\hat{\beta}_{\mathrm{GLS}}^{\mathrm{IV}}-\hat{\beta}_{\mathrm{w}}^{\mathrm{IV}}\right)^{-1}\left(\hat{\beta}_{\mathrm{GLS}}^{\mathrm{IV}}-\hat{\beta}_{\mathrm{w}}^{\mathrm{IV}}\right)
$$

can be constructed. Under the null, $c$ is distributed as a Chi-square statistic with $k$ degrees of freedom. Simple algebra using equation (4) shows that c can be written as

$$
c=\left(\hat{\beta}_{\mathrm{H}}^{\mathrm{IV}}-\hat{\beta}_{\mathrm{B}}^{\mathrm{IV}}\right) \cdot\left(\mathrm{V}_{\mathrm{H}}+\mathrm{V}_{\mathrm{B}}\right)^{-1}\left(\hat{\beta}_{\mathrm{H}}^{\mathrm{IV}}-\hat{\beta}_{\mathrm{B}}^{\mathrm{IV}}\right) .
$$

One advantage of the latter formulation of the test statistic is that the covariance matrix of the difference between the between and within estimators is easier to compute. While the $\operatorname{Cov}\left(\hat{\beta}_{\mathrm{GLS}}^{\mathrm{IV}} \cdot \hat{\beta}_{\mathrm{H}}^{\mathrm{IV}}\right)$ is equal to $\mathrm{V}_{\mathrm{W}}=\mathrm{V}_{\mathrm{GLS}}$ if $\hat{\beta}_{\mathrm{GLS}}^{\mathrm{IV}}$ is asymptotically efficient, the estimated difference of the covariance matrices may not be positive definite in small samples. This equivalent formulation of the Chi-square test statistic generalizes a result of Hausman-Taylor (1981) to allow for IV estimation.

To this point, I have considered instrument sets of the general form $\left[Q, 2, P, v^{B}\right]$. Now I turn my attention to the choice of $B$. An obvious choice for $B$ is $Z$ itself. Then $\bar{Z}-\left[Q_{v} Z, P Z\right]$. In other words, the instruments $Z$ are used twice: first as deviations from their time means and then as the time means themselves. This is essentially the Hausman-Taylor (HT) estimator 
discussed in Breusch, Mizon, and Schmidt (1989). However, since the values of $Z_{i t}$ are uncorrelated with the individual effects for each $\tau$ under $H_{0}$, then each of the $T$ NxL matrices $z_{t}$, where $z_{t}=\left[z_{1 t}^{\prime}, \ldots, z_{N t}^{\prime}\right]^{\prime}$, can be used as instruments for $X_{t}$. As a result, more instruments are available which cannot decrease the efficiency of the GIS-IV estimator. Under the null hypothesis that individual values of $\mathrm{Z}$ are uncorrelated with the individual effects for all values of $t$, then the instrument set $B-z^{*}$ provides more efficient estimates of $\beta$ where $z^{\star}$ is formed as in Breusch, Mizon, and Schmide (1989). That is, $Z$ is an NT $\times$ TL matrix:

$$
Z^{*}=\left[\begin{array}{ccc}
Z_{11} & \cdots & Z_{1 T} \\
Z_{11} & \cdots & Z_{1 T} \\
Z_{\mathrm{N} 1} & \cdots & Z_{\mathrm{NT}} \\
Z_{\mathrm{N} 1} & \cdots & Z_{\mathrm{NT}}
\end{array}\right] T \text { times }
$$

Note that $Q_{v} z^{\star}=0$ and $P_{v} z^{*}=z^{*}$. Hence, $\bar{z}-\left[Q_{v} Z, z^{*}\right]$ and $\hat{\beta}_{G L S}^{I V}$ constructed using $2^{*}$ will be more efficient than if constructed using $P_{v} Z$.

This suggests that the appropriate specification test should employ the more efficient GLS estimator to obtain greater power. However, this will not turn out to be the case. The next section considers the asymptotic efficiency for a particular class of data processes to illustrate the issue.

\section{Asymptotic Efficiency}

I consider data of the following form for the model described in equation 1:

$$
\begin{aligned}
& \mathrm{X}_{i t}=\gamma_{1} \mathrm{X}_{i t-1}+\nu_{i t} \\
& z_{i t}-\gamma_{2} \mathrm{Z}_{i t-1}+\eta_{i t} \\
& \underset{N \rightarrow \infty}{\operatorname{plim}\left(\frac{1}{N T} \nu^{\prime} \eta\right)-\Sigma_{\nu \eta}} \\
& \underset{N \rightarrow \infty}{\operatorname{plim}}\left(\frac{1}{N T} \nu^{\prime} \nu\right)=\Sigma_{\nu}
\end{aligned}
$$




$$
\begin{aligned}
& p \lim \left(\frac{1}{N T} \eta^{\prime} \eta\right)-\Sigma_{\eta} \\
& \left|\gamma_{1}\right|<1 \quad i-1,2
\end{aligned}
$$

where $\Sigma_{\nu}$ is a $k \times k$ positive definite matrix, $\Sigma_{\eta}$ is an LXL positive definite matrix and $\Sigma_{\nu \eta}$ is a $k \times L$ non-zero matrix. I will occasionally refer to $\Sigma_{x}$ and $\Sigma_{2}$, which equal $\frac{1}{1-\gamma_{1}^{2}} \Sigma_{\nu}$ and $\frac{1}{1-\gamma_{2}^{2}} \Sigma_{\eta}$, respectively. I assume that $\eta$ is

uncorrelated with $\epsilon$. However, it may be correlated with $a$, this correlation noted by

$$
\operatorname{plim}_{N \rightarrow \infty}\left(\frac{1}{N} z_{t}^{\prime} \alpha\right)-\Sigma_{z \alpha}
$$

where $\Sigma_{z \alpha}$ will be an $L \times 1$ zero vector under the null hypothesis and non-zero otherwise.

This is a particularly simple structure for the data generation process but it has the appealing property that as $\gamma_{1}$ increases from 0 towards 1 , an increasing fraction of the variance of the random variable is due to the variation across individuals. ${ }^{2}$ Since panel data are often slow moving over time, the performance of the specification test at high levels of $\gamma_{1}$ is of considerable interest. I exclude the possibility that $\gamma_{1}-1$. In the context of this model, $\gamma_{i}-1$ would mean that none of the explanatory variables (or instruments) have any "within" variation and I would be unable to estimate $\hat{\beta}_{W}^{\mathrm{IV}}$. A more general model would allow some variables to be non-stationary. However, the greater model complexity would obscure the essential results without adding much in the way of insights. Define the between estimator using the means of the instruments as $\hat{\beta}_{1}$ and the between estimator using $Z^{*}$ as $\hat{\beta}_{2}$. Under the null hypothesis, the asymptotic covariance of $\sqrt{\mathrm{N}} \hat{\beta}_{1}$ is given by

2 For example, the between variance for $X$ as a fraction of the cotal variance equals $\left(2 \sum_{t} a_{t}-T\right) / T^{2}$ where $a_{t}-\sum_{s=1}^{t} \gamma_{1}^{s-1}$. This fraction varies between $1 / T$ and 1 as $\gamma_{1}$ increases from 0 to 1 . 


$$
V_{1}=\sigma \frac{2}{u} \frac{T^{2}\left(2 \sum_{t} b_{t}-T\right)}{\left(\Sigma\left(a_{t}+b_{t}\right)-T\right)^{2}}\left[\sum \sum_{x z}^{-1} \sum_{z=}^{\prime}\right]^{-1}
$$

where $a_{t}=\sum_{1}^{t} \gamma_{1}^{s-1}$ and $b_{t}=\sum_{\mathbf{s}}^{t} \gamma_{2}^{s-1}$. The asymptotic covariance matrix for $\sqrt{\mathbb{N}} \hat{\beta}_{2}$ is given by

$$
\mathrm{V}_{2}=\sigma \frac{2}{\mathrm{u}}\left[\frac{\mathrm{FB}^{-1} \mathrm{~F}^{\prime}}{\mathrm{T}^{2}} \sum_{\mathrm{xz}} \sum_{z}^{-1} \sum_{\mathrm{xz}}^{\prime}\right]^{-1}
$$

where $F=\left[a_{T}+b_{1}-1 \quad a_{T-1}+b_{2}-1 \ldots a_{1}+b_{T}-1\right]$ and $B$ is the TxT matrix

$$
B=\left[\begin{array}{cccccc}
1 & \gamma_{2} & \gamma_{2}^{2} & \gamma_{2}^{3} & \ldots & \gamma_{2}^{T-1} \\
& & & & \\
\gamma_{2} & 1 & \gamma_{2} & \gamma_{2}^{2} & \cdots & \gamma_{2}^{T-2} \\
& & & \vdots & & \\
\gamma_{2}^{T-1} & \gamma_{2}^{T-2} & \gamma_{2}^{T-3} & \gamma_{2}^{T-4} & \cdots & 1
\end{array}\right]
$$

Consider local alternatives of the form $\sum_{z \alpha} \neq 0$ and $\sqrt{ } N \sum_{z \alpha} \rightarrow \psi<\infty$ as $N$ approaches $\infty$. Under the null hypothesis, the probability 1 imit (as $N \rightarrow \infty$ ) of $\hat{\beta}_{w}^{\text {IV }}-\hat{\beta}_{b}^{I V}$ is zero and $c$ is Chi-square with $k$ degrees of freedom. Under the alternative hypothesis, $c$ is distributed as a non-central Chi square random variable with $k$ degrees of freedom and non-centrality parameter $\delta$ where $\delta^{2}=$ $\bar{q}^{\prime} M^{-1} \bar{q}, \bar{q}$ is the probability limit of $\sqrt{N}\left(\hat{\beta}_{u}^{\text {IV }} \cdot \hat{\beta}_{b}^{\text {IV }}\right)$ and $M$ is the asymptotic covariance of $\sqrt{\mathrm{N}} q_{2}$ (see Scheffe (1959)). Let $\dot{q}_{i}$ equal $\dot{q}$ with $\hat{\beta}_{i}$ substituted for $\hat{\beta}_{b}^{\text {IV }}(i=1,2)$. The asymptotic biases for the two estimators using the different set of instruments are

$$
\bar{q}_{1}=\frac{T^{2}}{\sum\left(a_{t}+b_{t}\right)-T}\left[\sum \sum_{x z}^{-1} \sum_{z=}^{\prime}\right]^{-1} \sum_{x=2} \sum_{x=2}^{-1} \sum_{z \alpha}
$$

and 


$$
\dot{q}_{2}=\frac{T \cdot F B^{-1} e_{I}}{F B^{-1} F^{\prime}}\left[\sum_{x z} \sum_{z}^{-1} \sum_{x z}^{\prime}\right]^{-1} \sum_{x z} \sum_{z}^{-1} \sum_{z \alpha} .
$$

If $\gamma_{1}=\gamma_{2}$, it is straightforward to show that $v_{1}=v_{2}$ and that $\bar{q}_{1}-\bar{q}_{2}$ leading to the following proposition:

Proposition 1: Given the model in equations (10) - (11) and the assumption that $\gamma_{1}-\gamma_{2}$, the two estimators $\hat{\beta}_{1}$ and $\hat{\beta}_{2}$ are equally efficient asymptotically and the power of the specification test of the hypothesis that the instrumental variables are uncorrelated with the individual effects is unaffected by the choice of instrument set.

Proof: see appendix.

As $\gamma_{1}-\gamma_{2}$ diverges from zero, the variances and power of the specification test begins to differ. Since $v_{1}-v_{2}$ is positive definite, it would appear that the power of the specification test should increase using $z^{*}$ as the set of instruments. However, it will turn out that $\bar{q}_{1}$ may also be greater than $\bar{q}_{2}$ which will increase the power of the test using the means of $z$ as instruments. For $\gamma_{1}>0$, this result is formalized in the following proposition.

Proposition 2: If $\gamma_{1}>0$ and $\gamma_{2}-0$, then the asymptotic power of the test statistic using $\bar{z}$ as instruments is greater than the power using $Z^{*}$ as instruments.

Proof: see appendix.

I now turn my attention to the case where $\gamma_{1}-0$ and $\gamma_{2}>0$. Some tedious algebra shows that the asymptotic bias for the test statistic under the alternative hypothesis is greater when $\vec{Z}$ is used as the set of instruments. Again, the variance of the between estimator is greater when $\bar{z}$ is used. In this case it is difficult however to show that the power of the test is greater when $\bar{z}$ is used as the instrument set rather than $z^{*}$. In the simple case where $\mathrm{k}-\mathrm{L}=\mathrm{l}$ a grid search shows that the test statistic using $\bar{z}$ is more powerful than when $2^{*}$ is used. 
The increase in power is quite dramatic as illustrated in figure 1. Again, $k=L=1$ and the covariance of $Z$ and $\alpha$ is set equal to half the variance of 2 . At $\gamma_{2}-.8$ and $T=7$, the increase in the number of rejections is $41 \%$ (power equals .14 versus .10) and declines to $26 \%$ at $\mathrm{T}-16$ (power is .33 versus .26). At $\gamma_{2}-9$, the test using the mean of the instruments rejects nearly twice as often as when the instruments for each time period are used separately. Note that at $\gamma=.9$ and $I=7,80$ of the variation in the data occurs across individuals rather than for individuals across time. It is quite typical for many panel data applications to lose 80 of the variance in the data when using the fixed effects estimator.

F1gure 2 graphs the efficiency gains from using the means of the Instruments for $k=I-1$ when $T=5$ and $\gamma_{1}$ and $\gamma_{2}$ vary between .1 and .9 . As pointed out above, the tests perform equivalently when $\gamma_{1}-\gamma_{2}$ and the test using the means of the instruments performs better as the two autocorrelations move apart. However, the improvement is not dramatic with a maximum improvement of less than 32\%. This raises the issue of the performance of the tests in small samples. We turn our attention to this issue in the next section.

\section{Smal1 Sample Characteristics of the Test}

Specification test statistics in general have been criticized for having low power (e.g. Holly [1982], Newey [1985]). One might expect that the power of the test would deteriorate further as a result of the additional noise from the instrumenting of variables in $x$. To consider how well the test works in practice, I present results from a Monte Carlo experiment. I consider a simple model with $k=L-1$, set $\beta$ equal to 1 in equation (1) and take draws from a normal distribution for $x_{1 t}, Z_{1 t}, \epsilon_{1 t}$, and $\alpha_{1}$, each with mean 0 . The first three variables have variance 1 and $\alpha_{i}$ has variance $1 / T$. The covariance of $z$ 
and $\epsilon$ is zero while the other covariances vary from experiment to experiment. ${ }^{3}$ After generating the data, I compute the within and between estimates of $\beta$, their variances, and the Chi-square test (which has 1 degree of freedom). ${ }^{4}$ I repeat the process 1000 times for each model.

For the first set of results, I set $\gamma_{1}-\gamma_{2}=0$. With these assumptions, $\delta^{2}$ is given by the formula

$$
\delta^{2}-\psi^{2} \mathrm{~T}^{2}\left(\frac{\mathrm{T}-1}{2 \mathrm{~T}-1}\right)
$$

The asymptotic power of the test increases with more time periods, and with a higher correlation between the time means of the instruments and the individual effects. Note that the tests should perform equally well based on the results from the last section. Table 1 presents Monte Carlo results with $N-200$ and $T-5$. $\operatorname{Cov}\left(\mathrm{X}_{1 t}, \epsilon_{1 t}\right)-0.4$ and $\sigma_{z \alpha}$ and $\sigma_{\mathrm{zz}}$ vary from 0 to 0.06 and 0.1 to 0.7 respectively. The numbers in each cell show the fraction of times the null hypothesis is rejected due to $c$ in equation (9) exceeding the 58 critical value for a Chi-square random variable with 1 degree of freedom. The top number in each cell presents results using the mean of $\mathrm{Z}$ as the instrument set while the bottom number uses the set $z^{*}$. For future reference, call the first test statistic $c_{1}$ and the second statistic $c_{2}$. The first column in the table shows the computed size of the test. Note that neither of these tests has a computed size near 58 at very low levels of correlation between $Z$ and $X$. This is suggestive of the results of Nelson and Startz (1990a, 1990b) who have shown that the distribution of IV estimators diverges dramatically from the asymptotic distribution in the presence of poor instruments.

3 I have also experimented with varying the variance of $\alpha_{1}$. The results are not qualitatively different.

4 Equivalently, I could take the square root of the statistic and use the standard normal distribution. Constructing the experiment with one degree of freedom allows me to avoid issues of direction in defining the local alternatives which affect the power of the test. 
The remaining columns in table 1 show the power of the test in the face of increasing correlation of $z$ with $\alpha$. In nearly every case, the power of $c_{2}$ is higher than that of $c_{1}$. The increase in power can be significant, particularly with poor instruments. These results are striking given the number of Individuals in the data set $(\mathrm{N}-200)$ as well as the fact that $\mathrm{c}_{2}$ has the same distribution asymptotically as $c_{1}$, Clearly, in the case where $\gamma_{1}-$ $\gamma_{2}=0$, the main advantage of $c_{2}$ over $c_{1}$ lies in its performance in the presence of poor instruments.

These results show that in the case where asymptotically the two formulations of the ChI-square test should give equivalent results, the test statistic using the more efficient estimator is a more powerful test statistic. However, Proposition 2 states that in cases where $\gamma_{1}>0$ and $\gamma_{2}=$ 0 , then the test statistic using the less efficient estimator is more powerful. Table 2 presents Monte Carlo results in the case that $\gamma_{1}=0.9$ and $\gamma_{2}=0$. Recall that this implies that $80 \%$ of the variance in $X$ is lost when the fixed effects estimator is used. In all other respects, the model is the same as in the first experiment. Table 2 shows the power of the two test statistics. The clear advantage of $c_{1}$ over $c_{2}$ is evident here. While both test statistics have the correct size at moderate levels of correlation between $Z$ and $X$, the power of $c_{1}$ is greater than the power of $c_{2}$ in every case conditional on the alternative. The increase in power can be quite substantial even in the presence of good instrumental variables. The power of the test using the inefficient between estimator in the case where $\sigma_{x z}=.7$ and $\sigma_{z \alpha}=.18$ is .942 compared to a power of .791 when the efficient between estimator is used to construct the Chi-square test. This suggests that in cases where there is significant time variation for the instrumental variable while there is little time variation for the explanatory variable, one should use the inefficlent between estimator to construct the Chi-square statistic to 
test for correlation between the instrumental variables and the individual effects.

The final set of Monte Carlo results in Table 3 provides guidelines for generalizing the results of Propositions 1 and 2 along with the Monte Carlo results of Tables $I$ and 2 . In table 3, I fix the convariance of $Z$ and $\alpha$ at .09 , the covariance of $Z$ and $X$ at .70 and the covariance of $X$ and $\epsilon$ at .40 and vary $\gamma_{1}$ and $\gamma_{2}$ from 0 to .90 . In all cases where $\gamma_{1}>\gamma_{2}, c_{1}$ has higher power than $c_{2}$. Again, the increase in power can be quite dramatic $\left(e, g, \gamma_{1}-.9, \gamma_{2}\right.$ - .25). This suggests that where there is more time variation in the instrumental variables than in the explanatory variables, the test statistic should be computed using the inefficient between estimator. Where $\gamma_{1}$ equals $\gamma_{2}$, there is no clear result with both test statistics performing about the same, as Proposition I suggests they should. As $\gamma_{2}$ becomes Iarger than $\gamma_{1}$, it becomes more likely that the $c_{2}$ out performs $c_{1}$ though the improvement is not large until $\gamma_{2}$ is much greater than $\gamma_{1}$.

\section{Conclusion}

Testing for correlated individual effects has become increasingly important with the greater use of panel data sets. This paper shows that the type of specification test often employed in models where all the explanatory variables are considered exogenous carries over in a straight forward manner to models with endogenous explanatory variables. However greater attention must be pald to the quality of the Instruments used for the explanatory variables if the actual size and power of the test statistic is to correspond to the theoretical size and power.

The between estimator used in the specification test can be constructed with different sets of instruments. In many cases, a larger set of instruments leads to a more powerful test statistic. However, it is often the case that the more efflcient test statistic uses a reduced set of instruments for the between estimator. While the variance of the test statistic is driven 
up in this case, so is the asymptotic bias which can more than offset the increase in variance. Such a case happens when the explanatory variables are slow moving over time while the instruments are not. In this case, there is a distinct advantage to constructing the specification test using the less efficient estimator to take advantage of its greater asymptotic bias. 


\section{References}

Amelia, T, and T.E. MaCurdy, "Instrumental Variable Estimation of an Error Components Model," Econometrica, 54(1986): 869-881.

Baltagi, B.H. and Q. Li, "A Note on the Estimation of Simultaneous Equations with Error Components," Econometric Theory (1991), forthcoming.

Breusch, T. G. Mizon and P. Schmidt, "Efficlent Estmation Using Panel Data," Econometrica, 57(1989): 695-700.

Chamberlain, G.C. "Pane1 Data," in Handbook of Econometrics, Vo1. II, ed. Z. Griliches and M. Intriligator. Amsterdam: Elsevier, 1984.

Cornwe11, C., P. Schmldt, and D. Wyhowski, "Simultaneous Equations and Panel Data," Journal of Econometrics (1991), forthcoming.

Hausman, J.A. "Specification Tests in Econometrics," Econometrica, 46(1978): $1251-1271$.

Hausman, J.A. and W.E. Taylor, "Panel Data and Unobservable Individual Effects," Econometr1ca, 49(1981): 1377-1398.

Holly, A. "A Remark on Hausman's Specification Test," Econometrica, 50(1982): $749-760$.

Holtz-Eakin, D. "Testing for Individual Effects in Autoregressive Models," Journal of Econometrics 39(1988): 297.307.

Maddala, G.S. "The Use of Variance Components Models in Pooling Cross Section and Time Series Data," Econometrica, 39(1971): 341-358.

Mundlak, Y. "Empirical Production Function Free of Management Bias," Journal of Farm Economics, 43(1961): $44-56$.

Nelson, C. and R. Startz, "The Distribution of the Instrumental Variables Estimator and its t-Ratio When the Instrument is a Poor One, "Journal of Business 63(1990a): S125-S140.

Nelson, C. and R. Startz, "Some Further Results on the Exact Small Sample Properties of the Instrumental Variable Estimator, " Econometrica 58(1990b): 967-976.

Nerlove, M. "A Note on Error Components Mode1s," Econometrica, 39(1971): $383-396$.

Newey, $w$. "Generalized Method of Moments Specification Testing," Journal of Econometrics, 29(1985): 229-256.

Scheffe, H. The Analysis of Variance, New York: J. Wiley and Sons, 1959. 
Proof of Proposition 1: Let $\gamma_{1}-\gamma_{2}-\gamma<1$. Therefore $a_{t}-b_{t}$ and $B^{-1}$ is given by

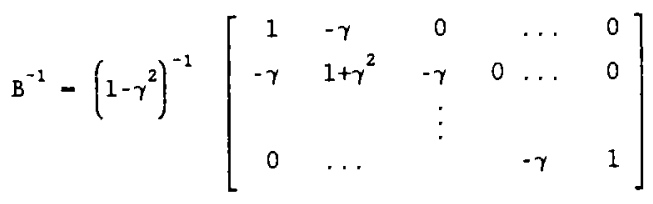

Then

$$
F B^{-1}=\left(1-\gamma^{2}\right)^{-1}\left[F_{1}-\gamma F_{2}\left(1+\gamma^{2}\right) F_{2}-\gamma\left(F_{1}+F_{3}\right) \ldots F_{T}-\gamma F_{T-1}\right]
$$

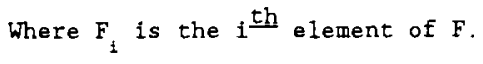

Since $a_{t}=\sum_{s=1}^{t} \gamma^{s-1}$, it is easy to show that $a_{t}-\gamma a_{t-1}=1$.

Therefore $F_{1}-\gamma F_{2}=a_{I}-\gamma\left(a_{I-1}+\gamma\right)$

$$
-1-\gamma^{2}
$$

Similarly $F_{\mathrm{T}}-\gamma \mathrm{F}_{\mathrm{T}-1}-1-\gamma^{2}$.

The expression $\left(1+\gamma^{2}\right) F_{T} \cdot \gamma\left(F_{t-1}+F_{t-1}\right), t-2, \ldots, T-1$ can be written as $-\gamma\left(a_{I-t+2}-\gamma a_{T-t+1}\right)+\left(a_{t}-\gamma a_{t-1}\right)+2 \gamma-1-\gamma^{2}+\left(a_{Y-t+1}-\gamma a_{Y-t}\right)-\gamma\left(a_{t+1}-\gamma a_{t}\right)-1-\gamma^{2}$

Therefore

$$
F B^{-1}=e_{T}^{\prime}
$$

$$
F B^{-1} F^{\prime}=2 \sum_{t=1}^{T} a_{t} \cdot T
$$

(A5)

$$
\mathrm{FB}^{-1} \mathrm{e}_{\mathrm{I}}=\mathrm{T}
$$

Substituting (A4) into equation (13) shows $v_{1}$ equals $v_{2}$ and substituting (A4) and (A5) into (15) shows $\bar{q}_{1}$ equals $\bar{q}_{2}$. 
Proof of Proposition 2: Define $\delta_{1}^{2}$ as the non-centrality paraneter for the test using $\bar{z}$ as the instrument set. Similarly, define $\delta_{2}^{2}$ for the instrument set $Z^{*}$. Let $V_{w}$ be the variance of the within estimator, First, I note that $\mathrm{V}_{1}-\mathrm{V}_{2}$ is a positive definite matrix, assuming $\gamma_{1}>0$ :

$$
V_{t}-V_{2}-T^{3} \sigma_{u}^{2}\left[\sum \sum_{x z}^{-1} \sum_{x z}\right]_{x z}^{-1}\left[\frac{1}{(\Sigma a)_{t}^{2}}-\frac{1}{T \Sigma a_{t}^{2}}\right]>0
$$

by Chebyshev's Inequality.

ג. Therefore,

$$
\text { It is easily shown that } \mathrm{v}_{1}^{-1} \overline{\mathrm{q}}_{1}-\mathrm{v}_{2}^{-1} \overline{\mathrm{q}}_{2}=\left(\Sigma \mathrm{a}_{\mathrm{t}} / \mathrm{T}\right) \sum_{\mathrm{x} z} \sum_{z z} \sum_{z \alpha}=
$$

$$
\delta_{1}^{2}-\delta_{2}^{2}-\lambda^{\prime}\left(\mathrm{R}_{1}-\mathrm{R}_{2}\right) \lambda
$$

and is greater than zero if $R_{1}-R_{2}$ is positive definite, where $R_{1}$ equals $\left[\mathrm{V}_{1}^{-1} \mathrm{~V}_{n} \mathrm{~V}_{1}^{-1}+\mathrm{V}_{1}^{-1}\right]^{-1}, \mathrm{i}-1,2 . \quad \mathrm{R}_{1}-\mathrm{R}_{2}$ will be positive definite if $\mathrm{R}_{2}^{-1}-\mathrm{R}_{1}^{-1}$ is positive definite. But

$$
\mathrm{R}_{2}^{-1}-\mathrm{R}_{1}^{-1}-\left(\mathrm{V}_{2}^{-1}-\mathrm{V}_{1}^{-1}\right) \mathrm{V}_{\mathrm{w}}\left(\mathrm{V}_{2}^{-1}-\mathrm{V}_{1}^{-1}\right)+\left(\mathrm{V}_{2}^{-1}-\mathrm{V}_{1}^{-1}\right)
$$

Each of the bracketed terms in $A 3$ is positive definite, so $R_{2}^{-1}-R_{1}^{-1}$ is positive definite and $\delta_{1}^{2}>\delta_{2}^{2}$. 
Table 1, Computed Power and Size

$$
\gamma_{1}=\gamma_{2}-0
$$

\begin{tabular}{|c|c|c|c|c|}
\hline & & & & \\
\hline & .0 & .02 & .04 & .06 \\
\hline .1 & $\begin{array}{l}.004 \\
.018\end{array}$ & $\begin{array}{r}.029 \\
.058\end{array}$ & $\begin{array}{l}.082 \\
.120\end{array}$ & $\begin{array}{l}.146 \\
.196\end{array}$ \\
\hline .3 & $\begin{array}{l}.032 \\
.044\end{array}$ & $\begin{array}{l}.170 \\
.184\end{array}$ & $\begin{array}{l}.457 \\
.475\end{array}$ & $\begin{array}{l}.754 \\
.744\end{array}$ \\
\hline .5 & $\begin{array}{l}.045 \\
.050\end{array}$ & $\begin{array}{l}.174 \\
.196\end{array}$ & $\begin{array}{l}.497 \\
.512\end{array}$ & $\begin{array}{l}.786 \\
.775\end{array}$ \\
\hline .7 & $\begin{array}{l}.051 \\
.063\end{array}$ & $\begin{array}{l}.181 \\
.205\end{array}$ & $\begin{array}{l}.459 \\
.489\end{array}$ & $\begin{array}{l}.803 \\
.807\end{array}$ \\
\hline
\end{tabular}

This table presents the fraction of rejections of the null hypothesis that $\sigma_{z a}=0$ out of 1000 replications. The top entry in each cell uses $\dot{z}$ as an instrument for $\bar{x}$ while the bottom entry uses $z^{*}$. The covariance of $X$ and $\epsilon$ equals .40, N equals 200 and $T$ equals 5 . The nominal size of the test is .05 . 
Table 2. Computed Power and Size

$$
\gamma_{1}=.9, \gamma_{2}=0
$$

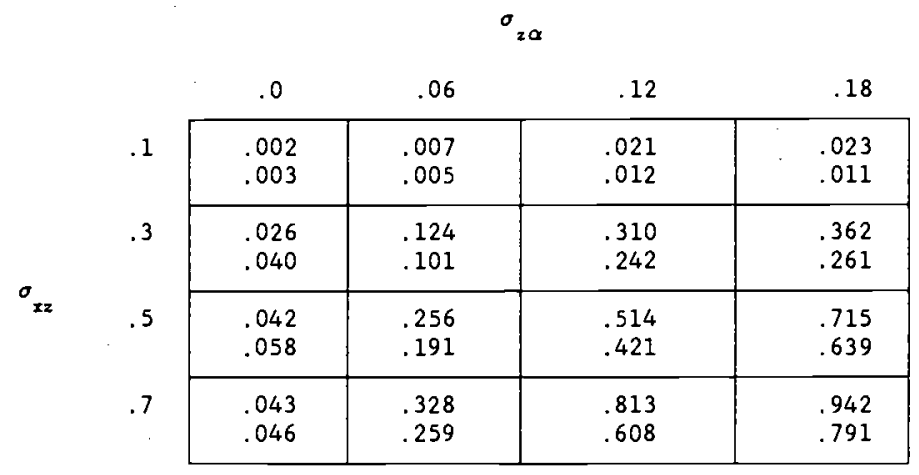

This table presents the fraction of rejections of the null hypothesis that $\sigma_{z \alpha}$ - 0 out of 1000 replications. The top entry in each cell uses $\dot{z}$ as an instrument for $\bar{X}$ while the bottom entry uses $z^{\star}$. The covariance of $X$ and $\epsilon$ equals $.40, \mathrm{~N}$ equals 200 and $\mathrm{T}$ equals 5 . The nominal size of the test is .05 . 
Table 3. Computed Power varying $\gamma_{1}$ and $\gamma_{2}$

\begin{tabular}{|c|c|c|c|c|c|}
\hline & .00 & .25 & .50 & .75 & .90 \\
\hline .00 & $\begin{array}{l}.979 \\
.980\end{array}$ & $\begin{array}{r}.967 \\
.962\end{array}$ & $\begin{array}{l}.942 \\
.929\end{array}$ & $\begin{array}{l}.837 \\
.748\end{array}$ & $\begin{array}{l}.606 \\
.430\end{array}$ \\
\hline .25 & $\begin{array}{l}.991 \\
.990\end{array}$ & $\begin{array}{l}.976 \\
.972\end{array}$ & $\begin{array}{l}.969 \\
.956\end{array}$ & $\begin{array}{l}.899 \\
.847\end{array}$ & $\begin{array}{l}.730 \\
.583\end{array}$ \\
\hline .50 & $\begin{array}{r}.987 \\
.983\end{array}$ & $\begin{array}{l}.981 \\
.978\end{array}$ & $\begin{array}{r}.984 \\
.973\end{array}$ & $\begin{array}{l}.913 \\
.877\end{array}$ & $\begin{array}{l}.806 \\
.695\end{array}$ \\
\hline .75 & $\begin{array}{l}.975 \\
.977\end{array}$ & $\begin{array}{l}.957 \\
.963\end{array}$ & $\begin{array}{l}.956 \\
.953\end{array}$ & $\begin{array}{l}.879 \\
.861\end{array}$ & $\begin{array}{r}.770 \\
.682\end{array}$ \\
\hline .90 & $\begin{array}{l}.848 \\
.961\end{array}$ & $\begin{array}{l}.854 \\
.938\end{array}$ & $\begin{array}{r}.814 \\
.926\end{array}$ & $\begin{array}{l}.745 \\
.821\end{array}$ & $\begin{array}{l}.550 \\
.670\end{array}$ \\
\hline
\end{tabular}

This table presents the fraction of rejections of the null hypothesis that $\sigma_{2 \alpha}=0$ out of 1000 replications. The top entry in each cell uses $\dot{Z}$ as an instrument for $\dot{X}$ while the bottom entry uses $Z^{*}$. The covariance of $X$ and $\epsilon$ equals .40, $\mathrm{N}$ equals 200 and $\mathrm{T}$ equals 5 . The nominal size of the test is $.05 . \sigma_{z a}=.09$ and $\sigma_{z x}-.70$. 


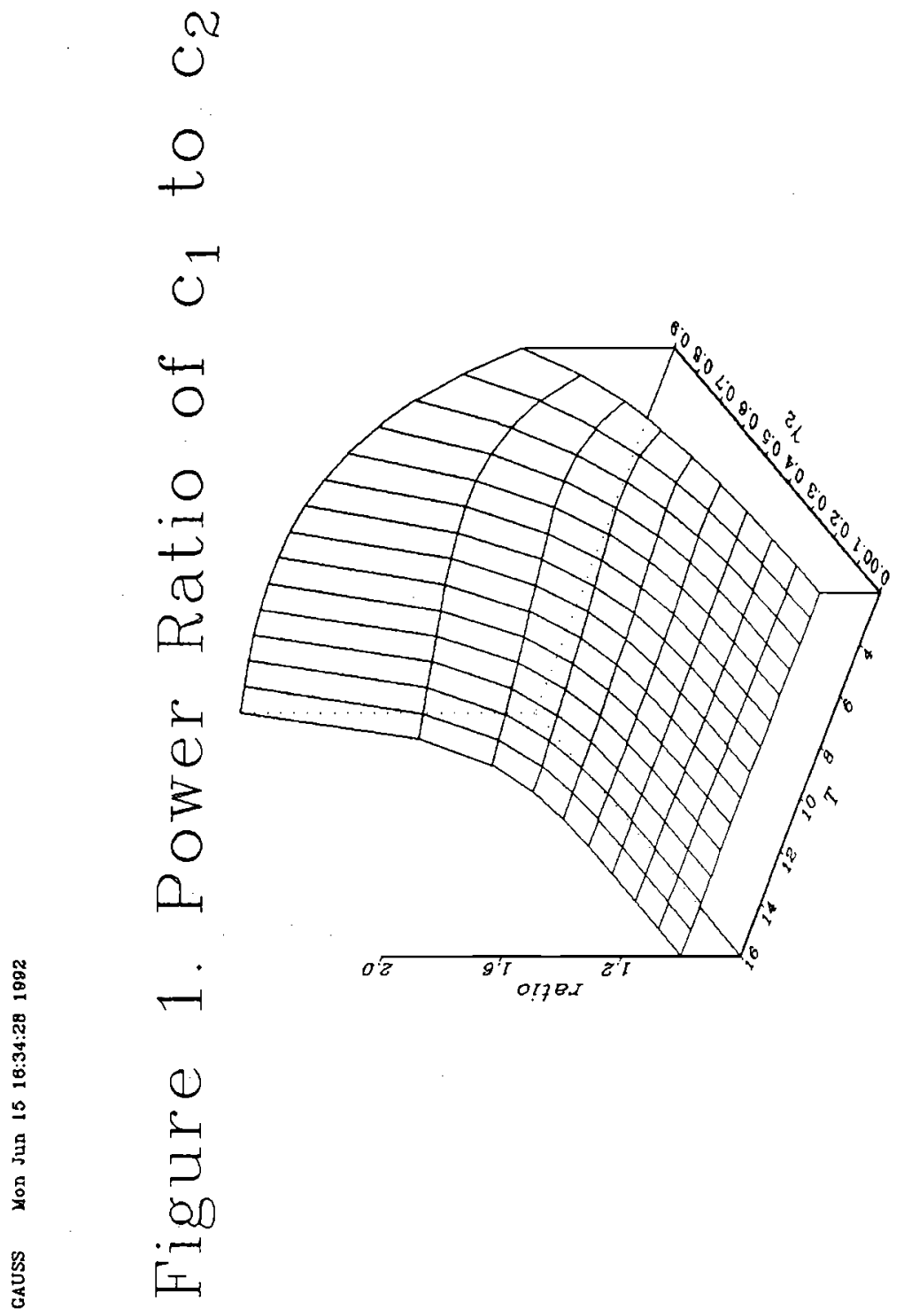

0
0
0
0
0 

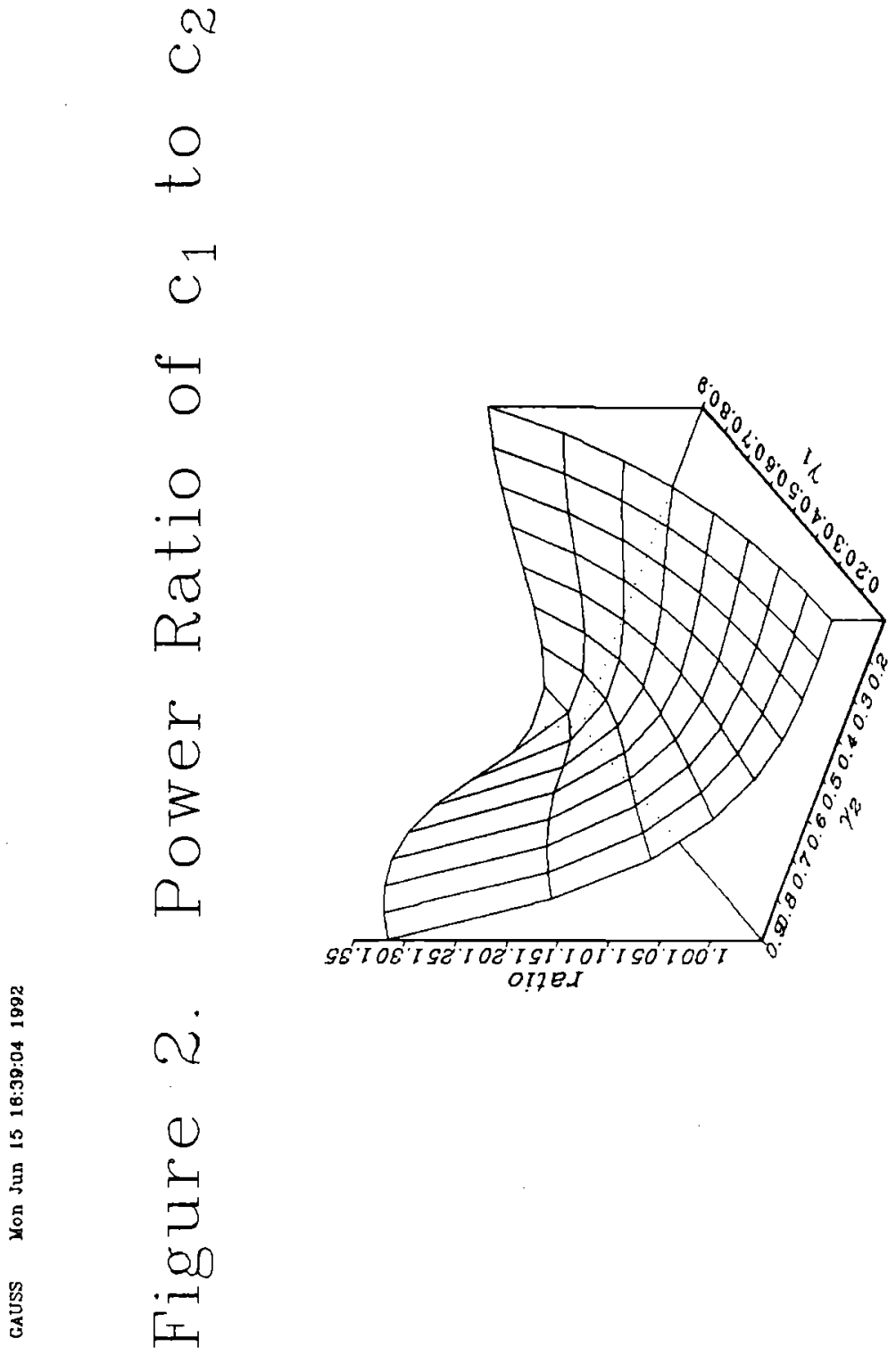

$\overline{0}$
0
0
$\vdots$
0 